\title{
Comparison of Planning Algorithm for Passive Optical Networks
}

\author{
Guojun Liu
}

China Electric Power Research Institute, No.15, Qing He Xiao Ying East Road, Haidian District, Beijing

100192, China

\section{Xin Wang}

School of Information and Communication Engineering, Beijing University of Posts and Telecommunications, No.10, Xi Tu Cheng Road, Haidian District, Beijing 100876,China

Yang Wang, Biyao Huang

China Electric Power Research Institute, No.15, Qing He Xiao Ying East Road, Haidian District, Beijing

100192, China

ABSTRACT: Though overviewing the recent researches in PON planning field, this paper mainly reviews the existing planning algorithms aiming to make a comparison of algorithms for PON. To this end, we could get a comprehensive understanding in the PON planning field and apply planning algorithm more efficiently. According to different standards, we classify the PON planning problems as different types. From the coverage span of PON, PON types can be classified as PON and long-reach PON; from the network convergence aspects, PON types can be classified as single PON and hybrid PON, such as hybrid WDM/TDM PON, TDM PON and convergence network of PON and WiMAX; from the numbers of ONUs, it can be classified as one hierarchy PON and multi-hierarchy PON; from the solution steps, it can be classified as one step approach and several suboptimal steps, which are allocation sub-problem and location sub-problem. Then we summarize all the schemes of PON planning, schemes of total deployment cost minimization, behavior-aware user assignment, QoS assurance and high availability or survivability guarantee. We make the performance comparison of planning algorithm for PON and use the specific parameters and numerous analysis. Taking certain constraints and conditions into account, we obtain the result that heuristic algorithm has got better performance than intuitive algorithm and moreover heuristic algorithm with some following-up researches and furthering optimization model apparently have been verified more effective than heuristic algorithm.

KEYWORD: PON planning; algorithm; performance comparison.

\section{INTRODUCTIONS}

As the rapid development of broadband access services, the FTTH (fiber to the home) networks as the key technology in Passive Optical Networks (PON) has been widely utilized and accelerating. Optical access networks provide the last mile service of the core network to the subscribers. PONs are currently being deployed as the optical access networks where an optical line terminal (OLT) at a remote node is connected to a group of subscriber terminals, namely Optical Network Units (ONUs) through fibers carrying the optical signal and a splitter splitting the optical signal into a number of equivalent signals propagating on distribution fibers to the ONUs. To efficiently planning for PON is essential for an economic and efficient resource utilized PON deployment, especially for rural areas. A proper network planning strategy that utilizes the minimal fibers layout can apparently increase the network profitability.

Since now, there have been some researches on PON planning considering various schemes. Cost minimization is the key objective and also quality of service (QoS) assurance and power aware optimization purpose for PON planning. We find the planning approaches, optimal planning models, novel PON architecture and heuristic algorithms have been proposed (Li, Ji 2009) (Gu, Rentao 2013) (Lv, Miao 2009) (Zukowski, Cezary 2014) (Kantarci, Burak, 2011).In this paper, we review all the recent researches in PON planning and classify various schemes. The primary purpose of this work is to extract existing approaches, and to compare their different performances and broaden the understanding of advances in this field.

The paper is structured as follows. In section 2, we elaborate the problem description of PON planning, classify the problem and summarize the schemes. In section 3, we summarize the 
optimization algorithms and make the performing comparison of algorithms. In section 4 , we make a brief conclusion of the paper.

\section{PROBLEM DESCRIPTION}

PON types can be classified in different standards, 1) from the coverage span of PON, PON types can be classified as PON ranged $20 \mathrm{~km}$ traditionally ( $\mathrm{Li}$, $\mathrm{Ji}$, 2009) (Agata,A.2012) and long-reach PON expanding to $100 \mathrm{~km}$ range $(\mathrm{Gu}$, Rentao, 2013) (Pal, S. 2014) (Kantarci, Burak, 2012) (Lin, Lin,2013) and there are two type of LR-PONs: "tree-andbranch" and "ring-and-spur" LR-PON(Shi, Lei, 2010); 2) from the network convergence aspects, PON types can be classified as single PON $(\mathrm{Gu}$, Rentao, 2013) (Pal, S. 2014) (Zukowski, Cezary 2014) (Lin, Lin,2013) and hybrid PON(Shi, Lei 2009) (Lv, Miao, 2009) (Shin 2010) (Lin, Bin, 2011), such as hybrid WDM/TDM PON(Shi, Lei 2009), TDM PON(Choi, Ki-Man, 2007), convergence network of PON and WiMAX (Lv, Miao 2010)(Shin, Dongseok, 2010) and cooperative network of PON and wireless (Lin, Bin, 2011); 3)from the numbers of ONUs, it can be classified as single-hierarchy PON and multi-hierarchy PON(Lv, Miao 2009); 4) from the solution steps, it can be classified as one step approach and several suboptimal steps, which is allocation sub-problem and location sub-problem, firstly cluster the optical distribution network and then plan the connection relationships of routing planning $(\mathrm{Li}, \mathrm{Ji}, 2009)(\mathrm{Lv}$, Miao 2009).

To achieve better performance in PON planning, not only the key objective of cost minimization, but also there are some existing researches concerning various schemes, as summarized below.

\subsection{Total deployment cost minimization scheme:}

It is concerned as a key optimal objective in every types of PON especially in long-reach PON. Generally PON cost is made up of some parts: the cost of optical devices, fiber cable and license cost (e.g., trenching and laying fiber cables), etc. The latter two parts overwhelms all the total costs in the whole deployment. Thus, we mainly minimize the total distances of fiber layout to achieve the goals.

\subsection{Behavior-aware user assignment and cost constrained scheme:}

In this scheme, there only has a few researches. Excepting for achieving the optimal goals of PON planning, also considering various network usage behaviors of different kind of users, such as users' daily usage profiles, we achieve the efficient network utilization by assign users elastic wavelengths efficiently. This scheme has been applied in hybrid WDM/TDM PON (Shi, Lei, 2009) and long-reach PON (Shi, Lei, 2010) so far.

\subsection{QoS assurance and cost constrained scheme:}

QoS assurance scheme is proposed in the convergence network of PON and WiMAX. They realize this by meeting high data rate and low packet error rate to wireless AP for various types of traffic (Shin, Dongseok, 2010).

\subsection{High availability or survivability guarantee and cost constrained planning scheme:}

High availability or survivability guarantee prevent huge data from losing in the last mile network of PON. This is scheme is proposed in long-reach PON (Kantarci, Burak, 2012).

\subsection{Modeling the optimization targets and constraints}

Generally the cost minimization can be modeled as bellows:

\section{Minimal objective function:}

Total cost of PON deployment mainly consists of three parts: cost of splitters, cost of fiber cables and cost of fiber laying and trenching. The mathematical model of minimal objective function is as equation (1):

$$
\begin{aligned}
& C_{t}^{s} \cdot \sum_{i \in \Omega_{C S}} S_{i t}+\sum_{i \in \Omega_{C S}}\left(C_{o}^{f}+C_{b}^{f}\right) \cdot d_{1 i}^{o c} \cdot l_{1 i} \cdot \eta_{1 i} \\
& +\sum_{i, j \in \Omega_{C S}}\left(C_{o}^{f}+C_{b}^{f}\right) \cdot d_{j i}^{c c} \cdot l_{j i} \cdot \eta_{j i} \\
& +\sum_{j \in \Omega_{C S}, k \in \Omega_{O N U}}\left(C_{o}^{f}+C_{b}^{f}\right) \cdot d_{j k}^{c o} \cdot l_{j}^{k} \cdot \eta_{j k}
\end{aligned}
$$

where $C_{t}^{s}=$ the cost of splitters; $C_{o}^{f}=$ the unit cost of fiber cables; $C_{b}^{f}=$ the average unit cost of fiber laying and trenching. And $\Omega=$ the set of PON devices, $S=$ the set of splitters, $d=$ the connection matrix between OLT and candidate sites, candidate sites and candidate sites and candidate sites to OUNs. $l=$ the distance parameters of $\mathrm{PON}, \eta=$ the weighted cost factor of path to path.

\section{Basic constraints:}

The type of splitters which consists of split ratio and optical power allocation ratio(even or uneven), connection relationship among OLT, ONUs and splitters, maximal distances between OLT to each ONU, maximal time delay between OLT to each ONU and practical PON topologies(cascaded ,trees or stars, etc.). 


\section{Special constraints:}

Behavior-aware and effective user assignment constraints, QoS assurance constraints and high availability or survivability guarantee constraints.

\section{OPTIMIZATION APPROACHES}

\subsection{Classifications of optimal algorithms}

Optimal algorithms for PON planning can generally fall into several categories: intuitive algorithm, heuristic algorithm, optimization model such as ILP or MILP, and mixed method of heuristic algorithm and optimization model.

\subsubsection{Intuitive algorithm}

Intuitive algorithm is generally simple and not concern comprehensive conditions. For example, there has been a research proposing an intuitive algorithm named as intuitive sectoring algorithm. It is very simple and similar to cutting a full cake. But some basic physical constraints of PON planning such as maximal transmission distance and maximal differential distance are not considered. Thus intuitive algorithm is generally not so precise and practically applicative (Li, Ji, 2009).

\subsubsection{Heuristic algorithm}

Generally, heuristic algorithm is extended from some nature phenomenon and existing algorithm to be improved. For example, the heuristic algorithm, named Recursive Allocation and Location Algorithm (RALA) is extended from Cooper's algorithm, which is used to solve the Multi-Facilities Location Problem (MFLP) in logistics studies (Cooper, L., 1964).From the simulation studies, heuristic algorithm is evaluated more efficient than intuitive algorithm ( $\mathrm{Li}, \mathrm{Ji}, 2009$ ). And it is also evaluated to be close to the enumeration method which is the precise approach (Gu, Rentao, 2013).

\subsubsection{Optimization model}

Integer Linear Programming (ILP) and mixed integer linear programming (MILP) are the common used optimization models. For example, MILP model for various survivability policies is deployed in reliable long-reach PONs under the budget limitations and evaluated as the most reliable protection policy compared to benchmark approach (Kantarci, Burak 2011).

\subsubsection{Mixed method of heuristic algorithm and optimization model}

Sometimes, pure heuristic algorithm may not reach the most optimal goal and still need to be improved, we need to further the research. Thus, after analysis by heuristic algorithm, we make the following-up research by optimization model. For example, the heuristic algorithm like RALA algorithm can find an efficient set of splitters. However based on this solution, according to the given parameter of the set of splitter, a Mixed Integer Linear Programming (MILP) model is employed to further optimize the connection relationship between each ONU and the splitters (Li, Ji, 2009).

To mention the above algorithm in detail, we make a specific description on the performance comparison of algorithms as bellows.

\subsection{Performance comparison of algorithms}

Usually, heuristic algorithm have the better performance than intuitive algorithm, for the latter method concerns less constraints and conditions than the former method, and intuitive algorithm has some unnecessary recursive steps. While to further optimize the planning problem, mixed method of heuristic algorithm and following-up optimization model should have better performance than pure heuristic algorithm. But from some existing researches, algorithm performance have the relationship with the size of network. For instance research (Li, Ji, 2009), for a medium-size PON, mixed method is a better solution than pure heuristic algorithm, while for a large-size PON,MILP optimization model may not be computable and pure heuristic algorithm saves $10 \%$ deployment costs.

Sometimes, we have to weigh the algorithm in different aspects, for instance $\operatorname{research}(\mathrm{Gu}$, Rentao, 2013)., if we only concern the accuracy performance, the proposed two-stage evolution algorithm performs quite close to precise solution in general scenarios and even got $0.1 \%$ error rate to the enumeration method. But if we concern the computation complexity, enumeration method has more computation time and waste more storage space, thus evolution algorithm is more effective than enumeration method which is more accuracy.

It has been verified that flexible multi-hierarchy PON planning brings more cost decrease economically than single hierarchy PON planning. Multi-hierarchy planning without routing optimization reduces $91.78 \%$ general cost than single-hierarchy planning. Multi-hierarchy planning with routing optimization reduces $30 \%$ general cost than multi-hierarchy planning without routing optimization (Lv, Miao 2009).

To choose small optical splitters near the endusers, deployment cost of fiber cables can be reduced, thus ILP formulations for LRPON reduce the initial deployment cost by more than $25 \%$ ( Zukowski, Cezary, 2014).

The specific parameters of algorithm performance comparison are shown in the Table 1. 
Table 1. The performance comparison of different algorithms.

\begin{tabular}{|c|c|c|c|}
\hline Reference Algorithm & Benchmark approach & Algorithm & Cost Saving $1 \%$ \\
\hline$(\mathrm{Li}, \mathrm{Ji}, 2009)$ & Intuitive algorithm & RALA & $50 \sim 70$ \\
\hline (Gu Rentao, 2013) & Enumeration method & Evolution algorithm & 98 \\
\hline (Lv Miao, 2009) & Single-hierarchy & Multi-hierarchy & 91.78 \\
\hline (Zukowski, Cezary, 2014) & Benchmark approach & ILP & 25 \\
\hline
\end{tabular}

\section{CONCLUSION}

In this paper, we overview recent researches in PON planning field and mainly make comparison of different algorithm performances. We conclude that generally intuitive algorithm has not got better performance than heuristic algorithm, because intuitive algorithm computed complex and use more storage space. While heuristic algorithm have some following-up researches and further optimize the problem, consequently got the effective performance. But we should also take certain constraints and conditions into account, because if some external factor changes like the coverage span of PON changed, heuristic algorithm may not always get the better performance. To achieve some special schemes, following-up investments have generally been verified more effective.

\section{REFERENCES}

[1] Agata, A \& Nishimura, K, 2012. Suboptimal PON network designing algorithm for minimizing deployment cost of optical fiber cables. $16^{\text {th }}$ International Conference on Optical Network Design and Modeling: 1-6.

[2] Choi,Ki-Man et al.2007. An Efficient Evolution Method from TDM-PON to Next-Generation PON. IEEE Photonics Technology Letters, 19(9):647-649.

[3] Cooper L.1964. Heuristic methods for location-allocation problems. SIAM Review, 6(1):37-53.

[4] Gu, Rentao et al. 2013. Evolutional Algorithm based cascade long reach passive optical networks planning. Communication in China 10:59-69.

[5] Kantarci, Burak, \& Mouftah H.T. 2011. Optimization models for reliable long-reach PON deployment.2011 IEEE Symposium on Computers and Communications $($ ISCC):506 - 511 .
[6] Kantarci, Burak \& Mouftah, H.T. 2012. Availability and Cost-Constrained Long-Reach Passive Optical Network Planning. IEEE Transactions on Reliability, 61(1):113124.

[7] Li, Ji \& Shen, Gangxiang. 2009. Cost Minimization Planning for Greenfield Passive Optical Networks. IEEE/OSA Journal of Optical Communications and Networking 1(1): 17-29.

[8] Lv, Miao \& Chen,Xue. 2009. Heuristic based multiHierarchy passive optical network planning. WICOM.2009:1-4.

[9] Lin,Bin et al. 2011. Dimensioning and Site Planning of Integrated PON and Wireless Cooperative Networks for Fixed Mobile Convergence. IEEE Transactions on Vehicular Technology, 60(9):4528-4538.

[10] Lin,Lin et al. 2013. Power-aware optimization modeling for cost-effective LRPON infrastructure deployment. 21st International Conference on Software, Telecommunications and Computer Networks (SoftCOM): 1-5.

[11] Lv,Miao \& Chen,Xue. 2010. A kind of planning algorithm for PON and WiMAX convergence_network. 2nd International Conference on Signal Processing Systems (ICSPS): V2:83-87.

[12] Pal, S. et al., 2014. Cable length minimisation in longreach-PON planning for sparsely populated areas. International Conference on Optical Network Design and Modeling: 234-239.

[13] Shi,Lei et al. 2010. Energy-Efficient Long-Reach Passive Optical Network: A Network Planning Approach Based on User Behaviors. IEEE Systems Journal, 4(4):449-457.

[14] Shi,Lei \& Song,Huan. 2009. Behavior-aware userassignment in hybrid PON planning. Optical Fiber Communication incudes post deadline papers. OFC 2009. Conference 1(3): 22-26.

[15] Shin, Dongseok et al.2010.Practical cell planning for internetworking between PON and WMN.2010 9th International Conference on Optical Internet (COIN):1-3.

[16] Zukowski, Cezary et al., 2014. Modelling accurate planning of PON nerworks to reduce initial investment in rural areas. International Conference on Optical Network Design and Modeling: 138-143. 